\title{
ON THE OUTER COEFFICIENT OF QUASICONFORMALITY OF A CYLINDRICAL MAP OF A CONVEX DIHEDRAL WEDGE
}

\author{
KARI HAG and MARJATTA NÄÄTÄNEN
}

\section{Introduction}

Let $D$ and $D^{\prime}$ be domains in $\boldsymbol{R}^{3}$ and let $f: D \rightarrow D^{\prime}$ be a homeomorphism. With each $f$ we can associate two numbers, the inner and outer dilatation of $f$,

$$
K_{I}(f)=\sup _{\Gamma} \frac{M(f \Gamma)}{M(\Gamma)}, \quad K_{o}(f)=\sup _{\Gamma} \frac{M(\Gamma)}{M(f \Gamma)},
$$

which measure how far $f$ is from being conformal. Here $M(\Gamma)$ and $M(f \Gamma)$ are the moduli of the curve families $\Gamma$ and $f \Gamma$, the suprema being taken over all families in $D$; see [6]. Further, the inner and outer coefficients of quasiconformality of $D$ with respect to $D^{\prime}$ are defined as

$$
K_{I}\left(D, D^{\prime}\right)=\inf _{f} K_{I}(f), \quad K_{O}\left(D, D^{\prime}\right)=\inf _{f} K_{O}(f) .
$$

The coefficients of quasiconformality have been calculated only for very few domains. For example, only $K_{O}\left(D, B^{3}\right)$ has been determined when $D$ is an infinite cylinder or an infinite convex cone. On the other hand, only $K_{I}\left(D, B^{3}\right)$ is known when $D$ is a convex dihedral wedge. More precisely, Gehring and Väisälä ([3], [6; p. 134]) have found that $K_{I}\left(D, D^{\prime}\right)=\beta / \alpha$ in the case of convex dihedral wedges with angles $\alpha, \beta$ and $\alpha \leqq \beta$. It is claimed by Syčev [4] that also $K_{o}\left(D, D^{\prime}\right)=\beta / \alpha$, but no proof is given. In Section 3 we show that this result follows easily for a subclass of mappings which satisfy a cylindrical condition; see 2.1. Taari [5] has obtained the same result for a subclass of mappings satisfying two local conditions; see 4.1. In Section 4 we use a mapping $f$ satisfying Taari's conditions to get a cylindrical map $h$ with $K_{o}(f) \geqq K_{o}(h)$. Hence Taari's result follows from our simpler argument.

\section{Basic notation}

2.1. Definitions. Let $(t, \psi, \varphi)$ be spherical coordinates in $\boldsymbol{R}^{3}$, where the polar angle $\varphi$ is measured from the positive $x_{3}$-axis. A domain in $R^{3}$ is a convex dihedral wedge of angle $\alpha, 0<\alpha \leqq \pi$, if it can be mapped by a similarity transformation onto the domain $D_{\alpha}=\{(t, \psi, \varphi) \mid t>0,0<\psi<\alpha, 0<\varphi<\pi\}$. 
Let $F$ denote the class of homeomorphisms $f: \bar{D}_{\alpha} \rightarrow \bar{D}_{\pi}, 0<\alpha \leqq \pi$, whose restrictions $f \mid D_{\alpha}: D_{\alpha} \rightarrow D_{\pi}$ are quasiconformal, and for sufficiently small radii map the intersection of $D_{\alpha}$ and a circular infinite cylinder with axis the $x_{3}$-axis onto the intersection of $D_{\pi}$ and a similar cylinder. We call such a mapping $f \in F$ cylindrical.

Let $F_{L}$ denote the class of homeomorphisms $f: \bar{D}_{\alpha} \rightarrow \bar{D}_{\pi}, 0<\alpha \leqq \pi$, whose restrictions $f \mid D_{\alpha}: D_{\alpha} \rightarrow D_{\pi}$ are quasiconformal, $f(0)=0$, and which satisfy the following condition at the origin:

$$
\lim _{\substack{\delta_{1} \rightarrow 0 \\ \delta_{2} \rightarrow 0}} \max r^{\prime}(x) / \min r^{\prime}(x)=1,
$$

where $\left(r^{\prime}, \psi^{\prime}, z^{\prime}\right)=f(r, \psi, z)$ in terms of cylindrical coordinates, and the maximum and minimum are taken over $x \in S\left(\delta_{1}, \delta_{2}\right)=\left\{(r, \psi, z)\left|r=\delta_{1},\right| z \mid \leqq \delta_{2}\right\} \cap \bar{D}_{\alpha}$. We call such a mapping $f \in F_{L}$ cylindrical at the origin.

2.2. Remark. We can state the definitions for closures of wedges without loss of generality since every cylindrical quasiconformal mapping $f: D_{\alpha} \rightarrow D_{\pi}$, $0<\alpha \leqq \pi$, can be extended to a homeomorphism $f^{*}: \bar{D}_{\alpha} \rightarrow \bar{D}_{\pi}$, such that $f^{*}(x) \rightarrow \infty$ as $x \rightarrow \infty$.

\section{The outer coefficient for cylindrical mappings}

3.1. Theorem. For the class $F$ of cylindrical mappings

$$
\inf \left\{K_{o}(f) \mid f \in F\right\}=\pi / \alpha .
$$

Proof. We show first that $K_{O}(f) \geqq \pi / \alpha$ for $f \in F$. Using cylindrical coordinates, let $r_{2}>r_{1}$ and $G_{i}=\left\{(r, \psi, z) \mid 0<r<r_{i}, 0<\psi<\alpha, z_{1}<z<z_{2}\right\}$ for $i=1,2$, and let $\Gamma$ be the family of curves joining the set $\left\{(r, \psi, z) \mid r_{1} \leqq r \leqq r_{2}, \psi=0, z_{1} \leqq z \leqq z_{2}\right\}$ to a similar set with $\psi=\alpha$, in the closure of $G_{2}-G_{1}$. Then as in Gehring [2; Lemma 1],

$$
M(\Gamma)=\left(r_{1}^{-1}-r_{2}^{-1}\right)\left(z_{2}-z_{1}\right) \alpha^{-2} .
$$

Let $\Gamma_{1}$ be the family of curves joining the sets $\left.\bar{G}_{1} \cap(r, \psi, z) \mid z=z_{i}\right\}, i=1,2$, in $\bar{G}_{1}$. Then by Väisälä [6; 7.2],

$$
M\left(\Gamma_{1}\right)=\alpha \pi r_{1}^{2} /\left(2 \pi\left(z_{2}-z_{1}\right)^{2}\right)
$$

By (1) and (2),

$$
M(\Gamma)^{2} M\left(\Gamma_{1}\right)=\frac{1}{2} \alpha^{-3}\left(1-r_{1} / r_{2}\right)^{2} .
$$

Let $r_{i}^{\prime}$ be the radius of $f\left(G_{i}\right), i=1,2$, and let $\bar{z}_{i}$ and $\underline{z}_{i}$ be the maximal and minimal $z$-coordinates in the $f$-image of the set $\bar{G}_{2} \cap\left\{(r, \psi, z) \mid z=z_{i}\right\} \quad i=1,2$, respectively. Denote by $\Gamma^{\prime}$ and $\Gamma_{1}^{\prime}$ the images of $\Gamma$ and $\Gamma_{1}$ under $f$. Then

$$
\begin{aligned}
& M\left(\Gamma^{\prime}\right) \leqq\left(1 / r_{1}^{\prime}-1 / r_{2}^{\prime}\right)\left(\bar{z}_{2}-\underline{z}_{1}\right) \pi^{-2}, \\
& M\left(\Gamma_{1}^{\prime}\right) \leqq \frac{1}{2} \pi\left(r_{1}^{\prime}\right)^{2}\left(\underline{z}_{2}-\bar{z}_{1}\right)^{-2},
\end{aligned}
$$


and

$$
M\left(\Gamma^{\prime}\right)^{2} M\left(\Gamma_{1}^{\prime}\right) \leqq \frac{1}{2} \pi^{-3}\left(1-r_{1}^{\prime} / r_{2}^{\prime}\right)^{2}\left(\bar{z}_{2}-\underline{z}_{1}\right)^{2}\left(\underline{z}_{2}-\bar{z}_{1}\right)^{-2}
$$

Next we consider $\bar{z}_{2}-\underline{z}_{2}$. We extend $f$ to a quasiconformal mapping $\hat{f}$ of $\boldsymbol{R}^{3}$ using the same foldings as Taari [5]: Let $g: D_{\alpha} \rightarrow D_{\pi}$ denote the folding given by $g(r, \psi, z)=$ $(r, \pi \psi / \alpha, z)$. Next we extend $f \circ g^{-1}: D_{\pi} \rightarrow D_{\pi}$ to a quasiconformal mapping $f_{1}: \boldsymbol{R}^{3} \rightarrow \boldsymbol{R}^{3}$ by reflection. Finally, let $f_{2}: \boldsymbol{R}^{3} \rightarrow \boldsymbol{R}^{3}$ be $f_{2}(r, \psi, z)=\left(r, \psi^{\prime}, z\right)$, where

$$
\psi^{\prime}=\left\{\begin{array}{l}
\alpha \psi / \pi \text { for } 0 \leqq \psi \leqq \pi \\
\alpha+(2 \pi-\alpha) \pi^{-1}(\psi-\pi) \text { for } \pi \leqq \psi \leqq 2 \pi
\end{array}\right.
$$

Then $\hat{f}=f_{1} \circ f_{2}^{-1}: \boldsymbol{R}^{3} \rightarrow \boldsymbol{R}^{3}$ is quasiconformal and $\hat{f} \mid \bar{D}_{\alpha}=f$. Lemma 8.1 of [3] applies to $\hat{f}^{-1} \mid\left\{(r, \psi, z) \mid 0<r<r_{2}^{\prime}\right\}$ followed by the map $h(r, \psi, z)=(t, \psi, \varphi)$ with $t=e^{z}$, $\varphi=\pi r /\left(2 r_{2}\right)$. (We can assume $f$ to be normalized in such a way that $f(0,0, z) \rightarrow+\infty$ as $z \rightarrow+\infty$.) We get

$$
0 \leqq \bar{z}_{2}-\underline{z}_{2} \leqq r_{2}^{\prime} A K_{I}\left(h \circ \hat{f}^{-1}\right),
$$

where $A$ is an absolute constant.

On the other hand, by (3) and (4),

$$
\alpha^{-3}\left(1-r_{1} / r_{2}\right)^{2} \leqq K_{O}(f)^{3} \pi^{-3}\left(\bar{z}_{2}-\underline{z}_{1}\right)^{2}\left(\underline{z}_{2}-\bar{z}_{1}\right)^{-2} .
$$

Letting $z_{2} \rightarrow \infty$ we get by (5)

$$
\alpha^{-3}\left(1-r_{1} / r_{2}\right)^{2} \leqq K_{O}(f)^{3} \pi^{-3} .
$$

From this with $r_{1} \rightarrow 0$ we see that $K_{O}(f) \geqq \pi / \alpha$. On the other hand, if $f$ is the cylindrical map

$$
f(r, \psi, z)=(r, \pi \psi / \alpha, \pi z / \alpha)
$$

we have equality so the bound is sharp.

The above result can be extended to locally cylindrical mappings as follows:

3.2. Theorem. For the class $F_{L}$ of mappings cylindrical at the origin,

$$
\inf \left\{K_{o}(f) \mid f \in F_{L}\right\}=\pi / \alpha .
$$

Proof. Since the mapping (6) is cylindrical at the origin it suffices, by Theorem 3.1, to show that for each $f \in F_{L}$ there exists a $g \in F$ such that $K_{O}(f) \geqq K_{O}(g)$. As in the proof of Theorem 3.1, each $f \in F_{L}$ can be extended to a quasiconformal mapping $\hat{f}: \boldsymbol{R}^{3} \rightarrow \boldsymbol{R}^{3}$. Next, let $\hat{g}_{n}: \boldsymbol{R}^{\mathbf{3}} \rightarrow \boldsymbol{R}^{\mathbf{3}}$ be the sequence defined by $\hat{g}_{n}(x)=$ $a_{n} f(x / n)$, where $a_{n}$ is chosen in such a way that $\left|\hat{g}_{n}\left(e_{1}\right)\right|=1$. Since $\hat{g}_{n}(0)=0$ we conclude by $[6 ; 19.4,20.5]$ that $\left\{\hat{g}_{n}\right\}$ is a normal family and there is a subsequence $\hat{g}_{j}$, $j \in J \subset N$, converging to a limit function $\hat{g}: \boldsymbol{R}^{3} \rightarrow \boldsymbol{R}^{3}$. The convergence is uniform on compact subsets of $\boldsymbol{R}^{3}$ and $\hat{g}$ is a homeomorphism since $\hat{g}(0)=0, \quad\left|\hat{g}\left(e_{1}\right)\right|=1$; see $[6 ; 21.3]$. By $[6 ; 37.2] \hat{g}$ is quasiconformal, $\hat{g}\left(D_{\alpha}\right)=D_{\pi}$, and $K_{O}(f) \geqq K_{o}(g)$, 
where $g=\hat{g} \mid \bar{D}_{\alpha}$. It follows that for each $r_{0}>0 \mathrm{~g}$ maps the compact sets $\left\{(r, \psi, z)\left|r=r_{0},\right| z \mid \leqq M\right\} \cap \bar{D}_{\alpha}$ into some cylindrical surface. Also, $g(0)=0$ and, by a simple topological argument, $g \in F$.

3.3. Remark. So far we have only considered the problem of determining $K_{o}\left(D_{\alpha}, D_{\beta}\right), 0<\alpha \leqq \beta<\pi$, for $\beta=\pi$. Composing $f: D_{\alpha} \rightarrow D_{\beta}$ with the standard folding (6), $\alpha$ replaced by $\beta$, we deduce that $K_{o}\left(D_{\alpha}, D_{\beta}\right)=\beta / \alpha$.

\section{Application}

As an application we construct in Theorem 4.3 a cylindrical map $h$ from Taari's functions and show that Taari's result follows from our result.

4.1. Taari's conditions. Taari [5] considers the subclass of homeomorphisms $f: \bar{D}_{\alpha} \rightarrow \bar{D}_{\pi}, 0<\alpha \leqq \pi, f(0)=0$, whose restrictions $f \mid D_{\alpha}$ are quasiconformal mappings onto $D_{\pi}$ and which satisfy the following conditions at the origin:

Condition A. There is a polar angle $\varphi_{0}, 0<\varphi_{0}<\pi / 2$ such that the limit

$$
\lim _{t \rightarrow 0+} f(t e) / t=k(e) \neq 0, \infty
$$

exists for every $e \in \bar{D}_{\alpha}$ with $0 \leqq\left(e, e_{3}\right) \leqq \varphi_{0}$, where $\left(e, e_{3}\right)$ denotes the acute angle between the vectors $e$ and $e_{3}$.

Condition B. For $e \in \bar{D}_{\alpha}$

$$
\lim _{\varepsilon \rightarrow 0+} \max \left(k(e), k\left(e_{3}\right)\right) / \min \left(k(e), k\left(e_{3}\right)\right)=1,
$$

where the maximum and minimum are over all vectors $e$ such that $\left(e, e_{3}\right)=\varepsilon$.

4.2. Theorem. Let $f$ be a mapping satisfying Taari's conditions stated in 4.1. Then there is a cylindrical map $h$ such that $K_{O}(f) \geqq K_{O}(h)$.

Proof. Given $f$ we construct, stepwise, a map $\hat{h}: \boldsymbol{R}^{3} \rightarrow \boldsymbol{R}^{3}$ whose restriction $h=\hat{h} \mid \bar{D}_{\alpha}$ will be cylindrical and satisfy $K_{o}(f) \geqq K_{O}(h)$.

The first step is carried out in [5]. Using Condition A Taari constructs a mapping $g: \bar{D}_{\alpha} \rightarrow \bar{D}_{\pi}, g(0)=0$, such that for each $e \in \bar{D}_{\alpha}, 0 \leqq\left(e, e_{3}\right) \leqq \varphi_{0}$, the restriction of $g$ to the ray $\{t e \mid t \geqq 0\}$ is linear, i.e. $g(t e)=\operatorname{tg}(e)$. Furthermore, using additional rotation and stretching we can assume $g\left(e_{3}\right)=e_{3}$. Also, $K_{o}(g) \leqq K_{o}(f)$.

Next the mapping $g$ is extended to a quasiconformal map $\hat{g}: \boldsymbol{R}^{3} \rightarrow \boldsymbol{R}^{3}$ as in the proof of Theorem 3.1. We observe, for later use, that if $0 \leqq\left(e, e_{3}\right) \leqq \varphi_{0}$, the restriction of $\hat{g}$ to the ray $\{t e \mid t \geqq 0\}$ is linear. Now, let $T_{n}$ denote translation by $n e_{3}$ and let $\hat{h}_{n}=T_{-n} \circ \hat{g} \circ T_{n}$. For $t \geqq-n, \hat{h}_{n}\left(t e_{3}\right)=T_{-n}\left(\hat{g}\left((t+n) e_{3}\right)\right)=t e_{3}$; hence $\left\{\hat{h}_{n}\right\}$ is a normal family and has a subsequence $\hat{h}_{j}, j \in J \subset N$, converging to a quasiconformal mapping $\hat{h}: \boldsymbol{R}^{3} \rightarrow \boldsymbol{R}^{3}$. The convergence is uniform on compact subsets of $\boldsymbol{R}^{3}, \quad \hat{h}\left(D_{\alpha}\right)=D_{\pi}$ and $K_{O}(h) \leqq K_{O}(g)$, where $h=\hat{h} \mid \bar{D}_{\alpha}$. Hence $K_{O}(h) \leqq K_{O}(f)$. 
To complete the proof we will show that if $C$ is a circular cylinder (domain) with axis the $x_{3}$-axis, then $\hat{h}(C)$ is a similar cylinder. For such a cylinder $C$ let $C_{j}$ be the cone determined by the circle $\partial C \cap\left\{x \mid x_{3}=0\right\}$ and the point $(-j) e_{3}$, and put $C_{j}^{\prime}=\hat{h}_{j}\left(C_{j}\right)$. By Theorem 3 of Gehring [1] we find that $\hat{h}(C)=\operatorname{Ker} C_{j}^{\prime}$ since $C=\operatorname{Ker} C_{j}$. Next we show that $\operatorname{Ker} C_{j}^{\prime}$ is a cylinder, i.e. that if $x_{0} \in \operatorname{Ker} C_{j}^{\prime}$, then $\left\{x_{0}+t e_{3} \mid t \in \boldsymbol{R}\right\} \subset \operatorname{Ker} C_{j}^{\prime}$. Let $\left(r_{0}, \psi_{0}, z_{0}\right)$ be the cylindrical coordinates of $x_{0}$ and let $x_{0} \in \operatorname{Ker} C_{j}^{\prime}$. Then there exists an $\varepsilon>0, \varepsilon<r_{0}$, such that the cylindrical neighborhood $N$ with $\left|r-r_{0}\right|<\varepsilon,\left|\psi-\psi_{0}\right|<\varepsilon,\left|z-z_{0}\right|<\varepsilon$ is in $C_{j}^{\prime}$ for $j \geqq j_{0}$. Next consider $C_{j}^{\prime}$. For large $j, \hat{g}$ maps $C_{j}+j e_{3}$ in such a way that each ray through the origin is mapped onto a ray through the origin. This means that $C_{j}^{\prime}$ is a union of rays through $-j e_{3}$; in particular, all rays through $-j e_{3}$ meeting $N$ are in $C_{j}^{\prime}$. In the case $t>0$ take $j$ so large that also $z_{0}+j>0$ and

$$
\arctan \frac{r_{0}-\varepsilon}{z_{0}+j+\varepsilon} \leqq \arctan \frac{\varepsilon / 2}{t-\varepsilon / 2} .
$$

Then the cylindrical neighborhood of $x_{0}+t e_{3}$ with $\left|r-r_{0}\right|<\varepsilon / 2,\left|\psi-\psi_{0}\right|<\varepsilon / 2$, $\left|z-\left(z_{0}+t\right)\right|<\varepsilon / 2$ is in $C_{j}^{\prime}$. Hence $\left\{x_{0}+t e_{3} \mid t \geqq 0\right\} \subset \operatorname{Ker} C_{j}^{\prime}$. Similarly, $\left\{x_{0}+t e_{3} \mid t<0\right\} \subset$ $\operatorname{Ker} C_{j}^{\prime}$. Finally we use Condition B to show that $\hat{h}(C)$ is circular with axis the $x_{3}$-axis. Since $k(e)=g(e)$, we see by Condition B that for all $j$

$$
\lim _{\delta \rightarrow 0+} \max \left(\hat{\mathrm{g}}\left(T_{j} x\right), e_{3}\right) / \min \left(\hat{\mathrm{g}}\left(T_{j} x\right), e_{3}\right)=1,
$$

where maximum and minimum are taken over all $x$ such that $\left(T_{j} x, e_{3}\right)=\delta$. Let $r>0$ and put $S=\left\{(r, \psi, z) \mid r=r_{0}, z=z_{0}\right\}$. For $j \geqq j_{1}$ we have by the above

$$
\max _{x \in S}\left(\hat{g}\left(T_{j} x\right), e_{3}\right) / \min _{x \in S}\left(\hat{g}\left(T_{j} x\right), e_{3}\right)<1+\varepsilon .
$$

The continuity of $\hat{g}$ at $e_{3}$, the linearity of $\hat{g}$ on rays close to the $x_{3}$-axis, and the fact that $\hat{g}\left(e_{3}\right)=e_{3}$ together imply that

$$
1-\varepsilon<\left|\hat{g}\left(x+j e_{3}\right)\right| /\left|x+j e_{3}\right|<1+\varepsilon
$$

for $j \geqq j_{2}, \quad x \in S$. Suppose that $j \geqq j_{1}, j_{2}$ and let $\underline{\varphi}_{j}=\min _{x \in S}\left(\hat{g}\left(T_{j} x\right), e_{3}\right)$, $\bar{\varphi}_{j}=\max _{x \in S}\left(\hat{g}\left(T_{j} x\right), e_{3}\right)$. By (7)

$$
\bar{\varphi}_{j}<(1+\varepsilon) \underline{\varphi}_{j} .
$$

From (8) we get for $x \in S$

$$
(1-\varepsilon) \sqrt{r_{0}^{2}+\left(z_{0}+j\right)^{2}}<\left|\hat{g}\left(x+j e_{3}\right)\right|<(1+\varepsilon) \sqrt{r_{0}^{2}+\left(z_{0}+j\right)^{2}} .
$$

Then by (9) and (10)

$$
\frac{\max _{x \in S} r\left(\hat{\mathrm{g}}\left(x+j e_{3}\right)\right)}{\min _{x \in S} r\left(\hat{\mathrm{g}}\left(x+j e_{3}\right)\right)}<\frac{(1+\varepsilon) \sin (1+\varepsilon) \underline{\varphi}_{j}}{(1-\varepsilon) \sin \underline{\varphi}_{j}} .
$$


Since $\hat{h}_{j}(x)=\hat{g}\left(x+j e_{3}\right)-j e_{3}$, we can replace $r\left(\hat{g}\left(x+j e_{3}\right)\right)$ by $r\left(\hat{h}_{j}(x)\right)$ in (11). Since $S$ is compact, $\hat{h}_{j} \rightarrow \hat{h}$ uniformly on $S$. Also, $\underline{\varphi}_{j} \rightarrow 0$ as $j \rightarrow \infty$, and the upper bound in (11) can be replaced by $(1+\varepsilon)^{3} /(1-\varepsilon)$. Since $\varepsilon>0$ was arbitrary,

for $x \in S$.

$$
\max r(\hat{h}(x)) / \min r(\hat{h}(x))=1
$$

\title{
References
}

[1] Gehring, F. W.: The Carathéodory convergence theorem for quasiconformal mappings in space. - Ann. Acad. Sci. Fenn. Ser. A I 336/11, 1963, 1-21.

[2] Gehring, F. W.: Extremal mappings between tori. - Amer. Math. Soc. Transl. (2) 104, 1976, $129-134$.

[3] Gehring, F. W., and J. VÄISÄLÄ: The coefficients of quasiconformality of domains in space. Acta Math. 114, 1965, 1-70.

[4] SyČEv, A. V.: The coefficients of quasiconformality of dihedral wedges. - Soviet Math. Dokl. 19, No. 2, 1978, 328-331.

[5] TAARI, O.: On the outer coefficient of quasiconformality of a convex dihedral wedge. - Ann. Acad. Sci. Fenn. Ser. A I 3, 1977, 215-221.

[6] VÄISÄL $\ddot{A}, J .:$ Lectures on $n$-dimensional quasiconformal mappings. - Lecture Notes in Mathematics 229, Springer-Verlag, Berlin-Heidelberg-New York, 1971.

\author{
University of Michigan \\ Department of Mathematics \\ Ann Arbor, Michigan 48109 \\ USA
}

University of Trondheim

Department of Mathematics, NTH

N-7034 Trondheim

Norway

Received 31 July 1979
University of Helsinki

Department of Mathematics

SF-00100 Helsinki 10

Finland 